\title{
THE
}

2-2-1998

\section{Universal Dynamics of Independent Critical Relaxation Modes}

M. P. Nightingale

University of Rhode Island, nightingale@uri.edu

H. W.J. Blöte

Follow this and additional works at: https://digitalcommons.uri.edu/phys_facpubs

Terms of Use

All rights reserved under copyright.

\section{Citation/Publisher Attribution}

Nightingale, M. P., \& Blöte, H. W.J. (1998). Universal Dynamics of Independent Critical Relaxation Modes. Physical Review Letters, 80(5), 1007-1010. doi: 10.1103/PhysRevLett.80.1007

Available at: http://dx.doi.org/10.1103/PhysRevLett.80.1007

This Article is brought to you for free and open access by the Physics at DigitalCommons@URI. It has been accepted for inclusion in Physics Faculty Publications by an authorized administrator of DigitalCommons@URI. For more information, please contact digitalcommons-group@uri.edu. 


\title{
Universal Dynamics of Independent Critical Relaxation Modes
}

\author{
M. P. Nightingale \\ Department of Physics, University of Rhode Island, Kingston, Rhode Island 02881 \\ H. W. J. Blöte \\ Department of Applied Physics, Delft University of Technology, P.O. Box 5046, 2600 GA Delft, The Netherlands \\ and Lorentz Institute, Leiden University, P.O. Box 9506, 2300 RA Leiden, The Netherlands
}

(Received 7 August 1997)

\begin{abstract}
We obtain the relaxation times of several, progressively rapid, independent modes of three models in a two-dimensional Ising universality class. Their size dependence can be described by one single dynamic exponent and universal amplitude ratios. This analysis is based on variational approximations of the eigenstates of the Markov matrix describing heat-bath, single-spin-flip dynamics. Monte Carlo computation of the corresponding autocorrelations and cross correlations, in which the variational error is systematically reduced, yields eigenvalues and the associated relaxation times with considerably higher statistical accuracy than is the case for traditional correlations. [S0031-9007(97)05176-4]
\end{abstract}

PACS numbers: 64.60.Ht, 02.70.Lq, 05.70.Jk, 64.60.Fr

It is generally accepted that static critical phenomena in two dimensions fall into classes characterized by universal critical exponents and amplitude ratios. However, for dynamic critical phenomena the situation is much less clear, because exact and accurate numerical results are scarce. In this Letter, we show that relaxation modes of a class, parameterized by $\kappa$, of two-dimensional Ising-like models with single-spin-flip dynamics have a universal exponent $z$ and universal amplitude ratios of the corresponding relaxation times. At critically, $\tau_{L i}(\kappa)$, the relaxation time of mode $i$ of a system of size $L$, behaves as

$$
\tau_{L i}(\kappa) \simeq m_{\kappa} A_{i} L^{z},
$$

where $A_{i}$ is universal, but depends on the mode $i$; the nonuniversal metric factor [1-3] $m_{\kappa}$ depends only on the microscopic details of the interactions and the dynamics. One is led to Eq. (1) by simple scaling arguments. These imply that self-similarity under spatial rescaling by a factor $b$ requires rescaling of time by $b^{z}$, and that similarity within a universality class is established by the metric factor which characterizes the time scale of each member of the class. Equation (1) is numerically verified by the Monte Carlo method introduced below.

This method is applied to square-lattice Ising models with nearest and next-nearest neighbor couplings $K$ and $K^{\prime}$, and periodic boundaries and Hamiltonian

$$
\mathcal{H} / k T=-K \sum_{(i, j)} s_{i} s_{j}-K^{\prime} \sum_{(i, j)^{\prime}} s_{i} s_{j},
$$

where $(i, j)$ and $(i, j)^{\prime}$ run over all nearest and nextnearest neighbor pairs. We choose three values of $\kappa=$ $K^{\prime} / K$, namely $\kappa=-\frac{1}{4}, 0,1$ : the opposite-, nearest-, and equivalent-neighbor models. The Markov matrix $P$ defines the dynamics. Element $P\left(S^{\prime}, S\right)$ is the conditional probability of a transition to $S^{\prime}$, given a configuration $S$. If $S$ and $S^{\prime}$ differ by more than one spin, $P\left(S^{\prime}, S\right)=0$. If they differ by precisely one spin, heat-bath probabilities apply:

$$
P\left(S^{\prime}, S\right)=\frac{1}{L^{2}}\left\{1-\tanh \left[\frac{\mathcal{H}\left(S^{\prime}\right)-\mathcal{H}(S)}{2 k T}\right]\right\},
$$

and $P(S, S)=1-\sum_{S^{\prime}} P\left(S^{\prime}, S\right)$. We denote by $1=$ $\lambda_{L 0}>\lambda_{L 1} \geq \ldots$ the eigenvalues of the Markov matrix. The associated relaxation times are $\tau_{L i}^{-1}(\kappa)=-L^{d} \ln \lambda_{L i}$. The dimensionality $d$ of the system enters because the Markov matrix evolves only one spin at a time.

We compute the spectrum of $P$ by means of a method used previously for a single eigenstate [4] generalized to several dominant eigenvalues of the Markov matrix. The second part of the method was introduced by Ceperley and Bernu in the context of quantum Monte Carlo methods [5]. Crucial in our approach is the construction of optimized trial states obtained by generalization of ideas of Umrigar et al. [6]. The optimization is applied to trial states of the following form. The leading eigenstate of the Markov matrix is the Boltzman distribution $\exp [-\mathcal{H}(S) / k T] \equiv \psi_{B}(S)^{2}$. Since $P$ satisfies detailed balance, $\tilde{P}\left(S^{\prime}, S\right) \equiv \psi_{B}\left(S^{\prime}\right)^{-1} P\left(S^{\prime}, S\right) \psi_{B}(S)$ is symmetric in $S$ and $S^{\prime}$ and equivalent to $P$. We approximate eigenvectors of $\tilde{P}$ by the functional form $\psi^{( \pm)}(S) \psi_{B}(S)$ defined in Eqs. (12) and (13) of Ref. [4]. That is, we construct translationally and rotationally invariant trial states, even or odd under spin inversion. These trial states are written as linear combinations of zero-momentum monomials in the magnetization and other long-wavelength Fourier components of the spin configuration.

We generalize to simultaneous optimization of multiple trial states, a powerful method [6-8] of optimizing a single many-parameter trial state: minimization of the variance of the configurational eigenvalue. Suppose that $\psi_{T}(S, p)$ is the value of the trial state $\psi_{T}$ for configuration $S$ and some choice of the parameters $p$ to 
be optimized. The configurational eigenvalue $\lambda(S, p)$ of a spin configuration $S$ is defined by

$$
\psi_{T}^{\prime}(S, p) \equiv \lambda(S, p) \psi_{T}(S, p),
$$

where the prime indicates matrix multiplication by $\tilde{P}$, i.e., $f^{\prime}(S)=\sum_{S^{\prime}} \tilde{P}\left(S, S^{\prime}\right) f\left(S^{\prime}\right)$ for arbitrary $f$. The optimal values of the variational parameters are obtained by minimization of the variance of $\lambda(S, p)$, estimated by means of a small Monte Carlo sample. The trial state, once optimized, is employed in a long Monte Carlo calculation yielding the corresponding eigenvalue of $\tilde{P}$. We refer to Ref. [4] for details and mention only a key feature of this method: for an (almost) exact eigenstate $\psi_{T}$, the variance of the eigenvalue (almost) vanishes. A similar zero-variance principle holds for the method of simultaneous optimization of several trial states to be discussed next.

For simplicity of presentation we first generalize the above method to a hypothetical case that yields $m$ exact eigenvalues of the Markov matrix $\tilde{P}$. Suppose we have $m$ basis states $\psi_{T i}, i=1, \ldots, m$ and again $M$ spin configurations $S_{\alpha}, \alpha=1, \ldots, M$ sampled from $\psi_{B}^{2}$. The case we consider is ideal in that we assume that these states $\psi_{T i}$ span an $m$-dimensional invariant subspace of $\tilde{P}$. In that case, by definition there exists a matrix $\hat{\Lambda}$ of order $m$ such that

$$
\psi_{T i}^{\prime}\left(S_{\alpha}\right)=\sum_{j=1}^{m} \hat{\Lambda}_{i j} \psi_{T j}\left(S_{\alpha}\right) .
$$

Again, the prime indicates matrix multiplication by $\tilde{P}$. If $M$ is large enough, $\hat{\Lambda}$ is for all practical purposes determined uniquely by Eq. (5) and one finds

$$
\hat{\Lambda}=\hat{N}^{-1} \hat{\mathcal{P}},
$$

where

$$
\begin{aligned}
& \hat{N}_{i j}=Z^{-1} \sum_{\alpha=1}^{M} \psi_{T i}\left(S_{\alpha}\right) \psi_{T j}\left(S_{\alpha}\right), \\
& \hat{\mathcal{P}}_{i j}=Z^{-1} \sum_{\alpha=1}^{M} \psi_{T i}\left(S_{\alpha}\right) \psi_{T j}^{\prime}\left(S_{\alpha}\right),
\end{aligned}
$$

and where $Z$ is an arbitrary normalization constant; again, the prime indicates matrix multiplication by $\tilde{P}$. In the nonideal case, the space spanned by the $m$ basis states $\psi_{T i}$ is not an invariant subspace of the matrix $\tilde{P}$. In that case, even though Eq. (5) generically has no true solution, Eqs. (6) and (7) still constitute solution in the least-squares sense, as may be verified by solving the normal equations.

If states span an invariant subspace, so does any nonsingular linear combination. In principle, the optimization criterion should have the same invariance. The spectrum of the matrix $\hat{\Lambda}$ has this property, which suggests that one subdivide the sample in subsamples and minimize the variance of the local spectrum over these subsamples. In practice, however, precisely this invariance gives rise to a near-singular nonlinear optimization problem. Therefore, to avoid slow or no convergence, we add a contribution to the above least-squares merit function to ensure that the basis states themselves are good approximate eigenstates, and we use an iterative optimization procedure: First a combination of the single and multieigenstate merit functions is used, and finally the resulting approximate eigenstates are optimized one at a time using the single-state procedure only. Unfortunately, this method is capricious and often we proceed by trial and error.

The variational states can be used directly only to obtain results with systematic errors, but these can be suppressed by the quantum Monte Carlo projection method introduced by Ceperley and Bernu [5]. Define generalized matrix elements

$$
\begin{gathered}
N_{i j}(t)=\left\langle\psi_{T i}\left|\tilde{P}^{t}\right| \psi_{T j}\right\rangle, \\
\mathcal{P}_{i j}(t)=\left\langle\psi_{T i}\left|\tilde{P}^{t+1}\right| \psi_{T j}\right\rangle .
\end{gathered}
$$

For $t=0$ Eqs. (7) are Monte Carlo estimators for these matrix elements, apart from the inconsequential normalization constant $Z$. One can view the matrix elements for $t>0$ as having been obtained by the substitution $\left|\psi_{T i}\right\rangle \rightarrow \tilde{P}^{t / 2}\left|\psi_{T i}\right\rangle$, which implies that spectral weight of "undesirable" subdominant states is reduced. The matrix elements in Eqs. (8) are the following equilibrium autocorrelation and cross-correlation functions of the Markov process generated by the matrix $P$ : $\left\langle\psi_{T i}\left(S_{0}\right) \psi_{T j}\left(S_{t}\right)\right\rangle$ and $\left\langle\psi_{T i}\left(S_{0}\right) \psi_{T j}^{\prime}\left(S_{t}\right)\right\rangle$, where $S_{0}$ and $S_{t}$ are spin configurations separated in time by $t$ single-spin flips.

It should be noted that in the limit of vanishing statistical error, each eigenvalue estimate obtained by the above method is bounded from above by the corresponding exact eigenvalue. The reader is referred to Ref. [5] for further details and references. The systematic error decreases for increasing projection time $t$ while the statistical error increases. An optimal intermediate $t$ has to be chosen, which yields biased estimators and some uncertainty in the statistical error estimates.

Of the three Ising-like models investigated here, the critical point is exactly known only for the nearest-neighbor model, where it occurs at $K=K_{c}(0)=\frac{1}{2} \ln (1+$ $\sqrt{2})$. The critical points of the two crossing-bond models $-K_{c}(1)=0.1901926807(2)$ and $K_{c}\left(-\frac{1}{4}\right)=$ $0.6972207(2)$-were determined elsewhere [9]. That analysis confirmed with a high precision that both crossing-bond models belong to the static Ising universality class.

Monte Carlo averages were taken over $1.2 \times 10^{8}$ spin configurations, for system sizes in the range $5 \leq L \leq$ 20. For the nearest-neighbor model these samples were separated by a number of Monte Carlo steps per spin equal to one for $L=5$ and increasing quadratically to ten for $L=20$. For the other systems these numbers where multiplied by the appropriate scale factors. These surprisingly short intervals are possible because the convergence of the eigenvalue estimates as a function of projection 
TABLE I. Universality of the dynamic exponent $z$. Results of least-squares fits for the dynamic exponent for three Isinglike models and for five distinct relaxation modes, identified in the first column: o $k$ refers to odd mode number $k$ and $e k$ refers to the corresponding even mode. Subsequent pairs of columns list $L_{0}$, the smallest system size included in the fit, and the resulting estimates of $z_{\kappa}$ for three ratios $\kappa=K^{\prime} / K$. Estimated errors are shown in parentheses. The numerical errors in $z$ tend to increase for the faster relaxation modes. This is due to the proximity of other subdominant eigenvalues of $P$, which affect the convergence as a function of the projection time $t$. To account for this effect, and for possible flaws in Eq. (9), two standard errors are quoted.

\begin{tabular}{|c|c|c|c|c|c|c|}
\hline & $L_{0}$ & $z_{-\frac{1}{4}}$ & $L_{0}$ & $z_{0}$ & $L_{0}$ & $z_{1}$ \\
\hline o1 & 4 & $2.163(6)$ & 4 & $2.1666(14)$ & 4 & 2.1659 (16) \\
\hline $\mathrm{o} 2$ & 5 & $2.165(6)$ & 6 & $2.171(4)$ & 8 & $2.171(4)$ \\
\hline o3 & 7 & $2.11(4)$ & 8 & $2.178(8)$ & 9 & 2.167 (18) \\
\hline $\mathrm{e} 2$ & 6 & $2.166(6)$ & 5 & $2.168(2)$ & 5 & $2.168(2)$ \\
\hline e3 & 8 & 2.17 (2) & 9 & 2.14 (4) & 8 & 2.19 (2) \\
\hline
\end{tabular}

time $t$ in Eqs. (8) is governed by lower-lying Markov matrix eigenvalues. These are much smaller than the largest odd eigenvalue, which usually determines the relaxation rate. For the system size $L=5$, the Monte Carlo results for the largest odd eigenvalues of the three models were compared with numerically exact results [4]. The consistency of both types of results confirms the validity of our numerical procedures.

As noted before for the largest odd eigenvalue of the nearest-neighbor model [4], the high statistical accuracy of the Monte Carlo estimates of the eigenvalue is due to the accuracy of the approximation of the eigenvector of the Markov matrix by the optimized trial states. The present Monte Carlo results for the largest odd eigenvalues of the nearest-neighbor models agree with those of Ref. [4]. The new data are based on statistical sample smaller by a factor of about 7 , but the current trial vectors had more variational freedom.

For finite system sizes $L$ we expect to the leading scaling behavior $\tau_{L} \sim L^{z}$. Following Ref. [4], we assume corrections proportional to even powers of $1 / L$ :

$$
\tau_{L i} \approx L^{z} \sum_{k=0}^{n_{c}} \alpha_{k i} L^{-2 k}
$$

where the series is truncated at order $n_{c}$. Although we cannot exclude other powers in $1 / L$, we have used Eq. (9) to analyze the Monte Carlo relaxation times.

Results of such fits with $n_{c}=3$ are presented in Table I. The smallest systems do not fit Eq. (9) well for this value of $n_{c}$. However, the residuals decrease rapidly when $L_{0}$, the smallest size included in the fit, is increased. The smallest acceptable value of $L_{0}$, as judged from the $\chi^{2}$ criterion, is also included in Table $\mathrm{I}$.

The estimates of $z$ obtained from the largest odd eigenvalues for the three models shown in Table I are in a good agreement mutually and also with the result $z=$ 2.1665 (12) of Ref. [4] for the nearest-neighbor model. Universality of $z$ has independently been confirmed by Wang and $\mathrm{Hu}$ [10], with a level of precision in the order of $10^{-2}$. The results for the largest odd eigenvalues are in agreement with those obtained for the other relaxation modes. Although the differences do occasionally amount to $3 \sigma$, we attribute these to imperfections of Eq. (9) and underestimation of the statistical errors of the eigenvalues themselves. Thus we interpret the data in Table I as a confirmation that dynamic universality applies to different models and modes of relaxation.

Correlation-time amplitudes were obtained from leastsquares fits using Eq. (9) with $z$ fixed at $\frac{13}{6}$, which happens to be close to the most accurate results in Table I. These amplitudes are in excellent agreement with Eq. (1) and determine the nonuniversal metric factors $m_{\kappa}$. Defining $m_{1} \equiv 1$, we found $m_{-\frac{1}{4}}=2.391 \pm 0.002$ and $m_{0}=1.5572 \pm 0.0005$. Table II shows results of the fits. Figure 1 is a semilogarithmic plot of the effective, size-dependent amplitudes $A_{L i}(\kappa) \equiv \tau_{L i} L^{-z} / m_{\kappa}$ derived from the spectral gaps of the Markov matrices of the opposite-, nearest, and equivalent-neighbor Ising models, $\kappa=-\frac{1}{4}, 0$, and 1 . The data collapse clearly illustrates the universality of the amplitude ratios. Finite-size dependences, clearly resolved in the fits, are only barely visible in the figure, but can be reconstructed by assuming $L^{-2}$ corrections.

We note that, if one suppresses all but the magnetization dependence of the optimized trial states, one obtains reasonably good approximate. Their number of

TABLE II. Universality of relaxation-time amplitudes. Results of least-squares fits for the finite-size amplitudes for three Isinglike models and for five distinct relaxation processes. The first column and ones labeled $L_{0}$ are as in Table I. The columns labeled $A_{i}(\kappa)$ contain the amplitudes defined in Eq. (1) for three interaction ratios $\kappa=K^{\prime} / K$ with metric factors $m_{\kappa}$ as given in the text. Estimated errors, as defined in Table I, are shown in parentheses. The difference $A_{i}(1)-A_{i}(\kappa)$ divided by its error is denoted by $r$.

\begin{tabular}{lclrrrrrr}
\hline \hline & $L_{0}$ & \multicolumn{1}{c}{$A_{i}\left(-\frac{1}{4}\right)$} & $r$ & $L_{0}$ & $A_{i}(0)$ & $r$ & $L_{0}$ & $A_{i}(1)$ \\
\hline o1 & 5 & $2.827(3)$ & 1.1 & 5 & $2.8318(8)$ & -0.6 & 5 & $2.8311(10)$ \\
e2 & 6 & $0.10503(2)$ & 0.1 & 5 & $0.10504(5)$ & 0.1 & 5 & $0.10504(2)$ \\
o2 & 5 & $0.04970(4)$ & -0.9 & 6 & $0.04958(2)$ & 1.6 & 8 & $0.04965(4)$ \\
e3 & 6 & $0.03009(5)$ & 0.3 & 9 & $0.03013(8)$ & -0.3 & 8 & $0.03011(6)$ \\
o3 & 6 & $0.01956(4)$ & -1.2 & 8 & $0.01955(4)$ & -0.9 & 9 & $0.01949(4)$ \\
\hline \hline
\end{tabular}




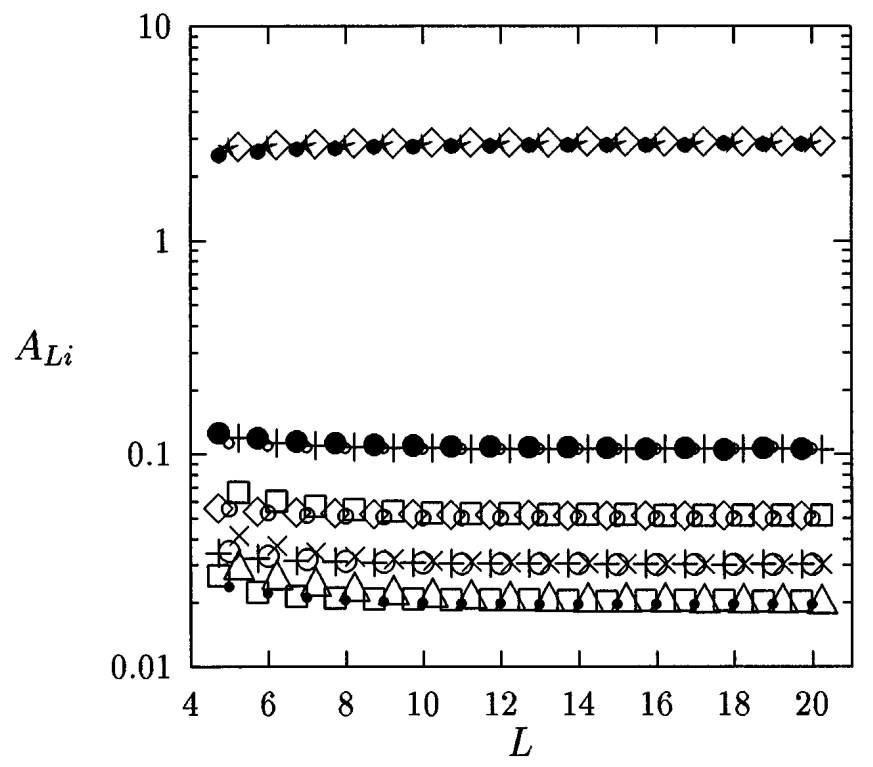

FIG. 1. Universality of relaxation-time amplitudes, shown by a plot of the effective, size-dependent amplitudes $A_{L i}$ on a logarithmic scale. To separate data points for the three models, $\frac{1}{4} \operatorname{sgn} \kappa$ was added to the abscissae. The data collapse predicted by Eq. (1) was produced by fitting two metric factors, $m_{0}$ and $m_{-\frac{1}{4}}$. Amplitudes of odd and even states alternate in magnitude.

nodes equals the number of the corresponding eigenvalue counted from the top of the spectrum, which is in agreement with the odd-even alternation shown in Table II. Thus, roughly speaking, one may associate the hiearchy of relaxation times with magnetization cumulants of increasing order. Since the second magnetization moment correlates strongly with the energy, the even cumulants apply to the relaxation of the energy as well. As an alternative interpretation of our results, we mention the following. The Markov matrix generates translations in time for dynamics. For statics, the transfer matrix generates the same in space and its spectrum matrix defines an infinite hierarchy of correlation lengths, with amplitudes satisfying the equivalent of Eq. (1) [11].

This research was supported by the (U.S.) National Science Foundation through Grants DMR-9725080 and
CHE-9625498 and by the Office of Naval Research. This research was conducted in part using the resources of the Cornell Theory Center, which receives or received major funding from the National Science Foundation (NSF) and New York State, with additional support from the Advanced Research Projects Agency (ARPA), the National Center for Research Resources at the National Institutes of Health (NIH), IBM Corporation, and other members of the center's Corporate Research Institute.

[1] M. P. Nightingale and H. W. J. Blöte, J. Phys. A 16, L657 (1983); see also references therein.

[2] V. Privman and M. E. Fisher, Phys. Rev. B 30, 322 (1984).

[3] H. W. J. Blöte and M.P. Nightingale, Physica (Amsterdam) 134A, 274 (1985).

[4] M. P. Nightingale and H. W. J. Blöte, Phys. Rev. Lett. 76, 4548 (1996).

[5] D. M. Ceperley and B. Bernu, J. Chem. Phys. 89, 6316 (1988). Also see B. Bernu, D. M. Ceperley, and W. A. Lester, Jr., J. Chem. Phys. 93, 552 (1990); W. R. Brown, W. A. Glauser, and W. A. Lester, Jr., J. Chem. Phys. 103, 9721 (1995).

[6] C. J. Umrigar, K. G. Wilson, and J. W. Wilkins, Phys. Rev. Lett. 60, 1719 (1988); C. J. Umrigar, K. G. Wilson, and J.W. Wilkins, in Computer Simulation Studies in Condensed Matter Physics, Recent Developments, edited by D. P. Landau, K. K. Mon, and H. B. Schüttler, Springer Proc. Phys. (Springer, Berlin, 1988).

[7] M. P. Nightingale, Computer Simulation Studies in Condensed Matter Physics, edited by D. P. Landau, K. K. Mon, and H. B. Schüttler, Springer Proc. Phys. (Springer, Berlin, 1997).

[8] M. P. Nightingale and C. J. Umrigar, in Recent Advances in Quantum Monte Carlo Methods, edited by W. A. Lester, Jr. (World Scientific, Singapore, 1997).

[9] M.P. Nightingale and H. W. J. Blöte, Physica A (Amsterdam) (to be published).

[10] F.-G. Wang and C.-K. Hu, Phys. Rev. E 56, 2310 (1997).

[11] M. P. Nightingale, in Finite-Size Scaling and Simulation of Statistical Mechanical Systems, edited by V. Privman (World Scientific, Singapore, 1990) pp. 287-351. 